\title{
Internal relaxation time in immersed particulate materials.
}

\author{
P. Rognon and I. Einar* \\ School of Civil Engineering, J05, The University of Sydney, Sydney, New South Wales 2006, Australia. \\ C. Gay \\ Matière et Systèmes Complexes, Université Paris Diderot-Paris 7, CNRS, UMR 7057, \\ Bâtiment Condorcet, Case courrier 7056, F-75205 PARIS cedex 13, France.
}

(Dated: October 30, 2018)

\begin{abstract}
We study the dynamics of the solid to liquid transition for a model material made of elastic particles immersed in a viscous fluid. The interaction between particle surfaces includes their viscous lubrication, a sharp repulsion when they get closer than a tuned steric length and their elastic deflection induced by those two forces. We use Soft Dynamics to simulate the dynamics of this material when it experiences a step increase in the shear stress and a constant normal stress. We observe a long creep phase before a substantial flow eventually establishes. We find that the typical creep time relies on an internal relaxation process, namely the separation of two particles driven by the applied stress and resisted by the viscous friction. This mechanism should be relevant for granular pastes, living cells, emulsions and wet foams.

PACS numbers: $82.70 .-\mathrm{y}, 83.80 . \mathrm{Iz}, 02.70 . \mathrm{Ns}$,
\end{abstract}

\section{INTRODUCTION}

The rheological behavior of soft amorphous materials such as foams [1, 2], emulsions [3, 4, [5], living cells [6] and granular pastes [7, 8] is an active field of research with many practical issues, for these materials are broadly involved in geophysical events, industrial processes and biological organisms. Besides, understanding their dynamics presents great theoretical challenges as their structure, similar to that of glasses, is disordered, metastable and out of thermodynamical equilibrium [9, 10].

Perhaps the most salient property of these materials is their ability to deform like a solid or to flow like a liquid depending on the loading conditions. At the microscopic level, this solid-liquid transition relies on the particles ability to move with respect to each other, similar to the cage effect in molecular or colloidal systems [11, 12]. However, large enough bubbles, droplets and grains do not show significant Brownian motion and their solidliquid transition is thus not thermally activated. The driving force of the relative particle motion comes only from the external loading, while the resisting force comes from the particle interactions and/or deformations. This force balance defines internal dynamics with an associated time-scale, which is the core of the thixotropy effects in various materials 12, 13, 14, 15, 16] and of the relaxation time evidenced by oscillatory shear experiments [17, 18]. The interstitial liquid is pivotal to these internal dynamics, as it affects both the driving force and the resisting force that the particles experience.

Immersed particulate materials support the stresses not only through their contact network (known as os-

\footnotetext{
*Electronic address: i.einav@usyd.edu.au

${ }^{\dagger}$ Electronic address: cyprien.gay@univ-paris-diderot.fr
}

motic or effective stresses), but also by the continuous liquid (pore pressure). This notion of pore pressure is a central point of soil stability analysis (see [19] and reference therein), and was shown to be responsible for a long delay in the triggering of submarine granular avalanches [20]. The underlying mechanism involves the dilatancy needed to start shearing and the time required to fill the growing pores with liquid. This filling time then relies on the degree of drainage of the shearing zone, in other word on how long the liquid takes to flow from one part of the material to another. Assuming that the granular skeleton does not deform, the flow of liquid through the pore network satisfies a Darcy law with some permeability [20].

Besides the pore pressure, the liquid has a strong effect on the particle interactions as it lubricates close pairs of surfaces. The viscous friction between two smooth rigid spheres is a well known problem (see for instance 21]). However, the presence of asperities on rough surfaces has been shown to strongly affect lubrication, and this topic is still an active field of research [22, 23, 24, 25]. Between bubbles or droplets, the flow of liquid is even more complex, as the surfaces are soft and can stretch 26, 27], they can be permeable [28] and exhibit some repulsive electrostatic interactions leading to disjoncting pressure [29] as well as adhesion [2, 30, 31].

This paper presents a numerical investigation on the dynamics of the solid-liquid transition in a model immersed particulate material. Our motivation is to capture the common characteristics of bubbles, droplets or solid grains: all of these particles can deform elastically and interact through lubricated contacts. Therefore, we do not address the flow of the liquid in the pore network or any of the others interactions mentioned above. Instead, here we focus on elastic spheres interacting via lubricated contacts with a steric repulsion when surfaces get very close. We simulate the dynamics of this material using Soft Dynamics, the discrete element method that 
we introduced in Refs. 32, 33]. We subject large samples to a constant normal (osmotic) stress and a (smoothed) stepwise increase of the shear stress. We then measure the delay before the onset of a steady material flow. Finally, we show that this macroscopic delay reflects the time taken by two particles for separating, a motion driven by a tensile force that results from the applied stress and that is resisted by the normal viscous friction.

\section{INTERNAL DYNAMICS: INSIGHTS FROM DIMENSIONAL ANALYSIS}

Dimensional analysis is often used to define the typical basic times that the system involves. Let us recall its outcome, as expressed in various studies. We are dealing with elastic particles of size $d$, Young's modulus $E$ and mass $m$. They are immersed in a Newtonian fluid (viscosity $\eta$ ) and confined by an osmotic normal stress $\sigma_{y y}$. Four time scales emerge from those quantities.

The first two time scales correspond to the motion of a single particle which experiences a driving force $\sigma_{y y} d^{2}$. For dry grains, this motion is resisted only by the grain inertia (mass $m$ ), while for particles with negligible mass immersed in a fluid of viscosity $\eta$, it is resisted merely by the Stokes drag. The respective corresponding times are the inertial time $t_{i}$ 34, 35, 36] and the Stokes time $t_{S t}$ [37, 38]:

$$
\begin{aligned}
t_{i} & =\sqrt{\frac{m}{\sigma_{y y} d}} ; \\
t_{S t} & =3 \pi \frac{\eta}{\sigma_{y y}} .
\end{aligned}
$$

The other two time scales can be directly obtained by replacing the scale of stress $\sigma_{y y}$ by the Young modulus $E$ in each above time scale. This leads to the time $t_{c}$ for sound to propagate through a grain (which can also be linked to the binary collision time [39]) and to the time $t_{M}$ for an elastically deformed particle to recover its shape when immersed in a viscous fluid, a time similar to that of a Maxwell relaxation [40, 41]:

$$
\begin{aligned}
t_{c} & =\sqrt{\frac{m}{E d}} \\
t_{M} & =\frac{\eta}{E}
\end{aligned}
$$

Tab. Igives an estimate of those time scales for elastic grains and for bubbles. For the particle deformation to remain moderate, the reduced normal stress $\kappa=\frac{\sigma_{y y}}{E}$ must be small, and time scale $t_{c}$ (resp. $t_{M}$ ) is thus shorter than $t_{i}$ (resp. $t_{S t}$ ).

The above references clearly show the usefulness of such time scales, especially for expressing flow laws. However, none of them accounts for the details of the interaction of a particle with its neighbourhood. The aim of the present paper is to point out the importance of such a time scale when particles are elastic and interact via lubricated contacts.
TABLE I: Order of magnitudes of the internal time scales in immersed particulate systems. Time scales from dimensional analysis (defined in section $\amalg$ ) and delay time scale $\Delta$ from lubrication interactions (computed through the present study). Particles consist in grains or bubbles of size $d=1 \mathrm{~mm}$ immersed in water (viscosity $10^{-3} \mathrm{Pas}$ ). The grain density is $2.10^{3} \mathrm{~kg} \mathrm{~m}^{-3}$ and their Young modulus is $10^{10} \mathrm{~Pa}$. The density of bubbles is null and their effective Young modulus is of the order of $E \approx 10^{2} \mathrm{~Pa}$. We consider an osmotic normal stress of $10^{7} \mathrm{~Pa}$ for the grains and $10^{-1} \mathrm{~Pa}$ for the bubbles in order to obtain the same reduced normal stress $\kappa=\sigma_{y y} / E=10^{-3}$ in both cases. The delay $\Delta$ is estimated according to Eq. (8) with a steric length of $h_{0}=10^{-7} \mathrm{~m}$, which corresponds to particles in the Hertz limit.

\begin{tabular}{lccccc}
\hline \hline Time $(\mathrm{s})$ & $t_{i}$ & $t_{S t}$ & $t_{c}$ & $t_{M}$ & $\Delta$ \\
\hline Grains & $10^{-5}$ & $10^{-9}$ & $10^{-7.5}$ & $10^{-13}$ & $10^{4} t_{S t}$ \\
\hline Bubbles & 0 & $10^{-1}$ & 0 & $10^{-5}$ & ${ }^{a} 10^{4} t_{S t}$ \\
\hline \hline
\end{tabular}

${ }^{a}$ The above delay $\Delta$ was based on Hertzian elasticity. Of course, bubbles and droplets do not display any bulk elasticity, yet they do recover their shape upon load reversal. For the present purpose, their effective Young modulus was estimated as the surface tension $\left(10^{-1} \mathrm{~N} \mathrm{~m}^{-1}\right.$ ) divided by the bubble size: $E \approx 10^{2} \mathrm{~Pa}$ (see [26] for more details on droplet contact).

\section{INTERNAL DYNAMICS: PARTICLE INTERACTION}

We consider an ideal suspension made of elastic particles in a Newtonian fluid. We focus on dense configurations, where the surface-to-surface distance $h$ between a particle and its few closest neighbours is typically much smaller than the particle diameter $d$. Under such conditions, the particle-fluid-particle interaction is mostly governed by the liquid that is present within a small gap of thickness $h$ and radius $a$ with $h \ll a \ll d$ (Fig[1a). Accordingly, the force and torque transmitted from surface to surface within each pair of neighbouring particles are proportional to their relative velocity. For such a gap with a low aspect ratio $(h \ll a)$ in which the flow satisfies the conditions of the lubrication approximation, the proportionality coefficients (which we here call viscous frictions) can be expressed rather easily (see Appendix A) as functions of the gap thickness $h$ and size $a$.

As a result of the viscous force and torque transmitted through the gap, the particle surfaces locally deform elastically. Hence, for each pair of close neighbours, the combination of the gap viscous response and the particle elasticities is analogous to a Maxwell element (see Fig 1, part a1) in which both the stiffness and the viscous friction are non-linear (in the sense that they depend on the gap geometry) and tensorial (since they reflect the normal, tangential and twist modes, see Fig. 51).

The viscous friction term that represents the normal relative motion (Poiseuille flow) turns out to be typically much higher than the corresponding terms for the tan- 

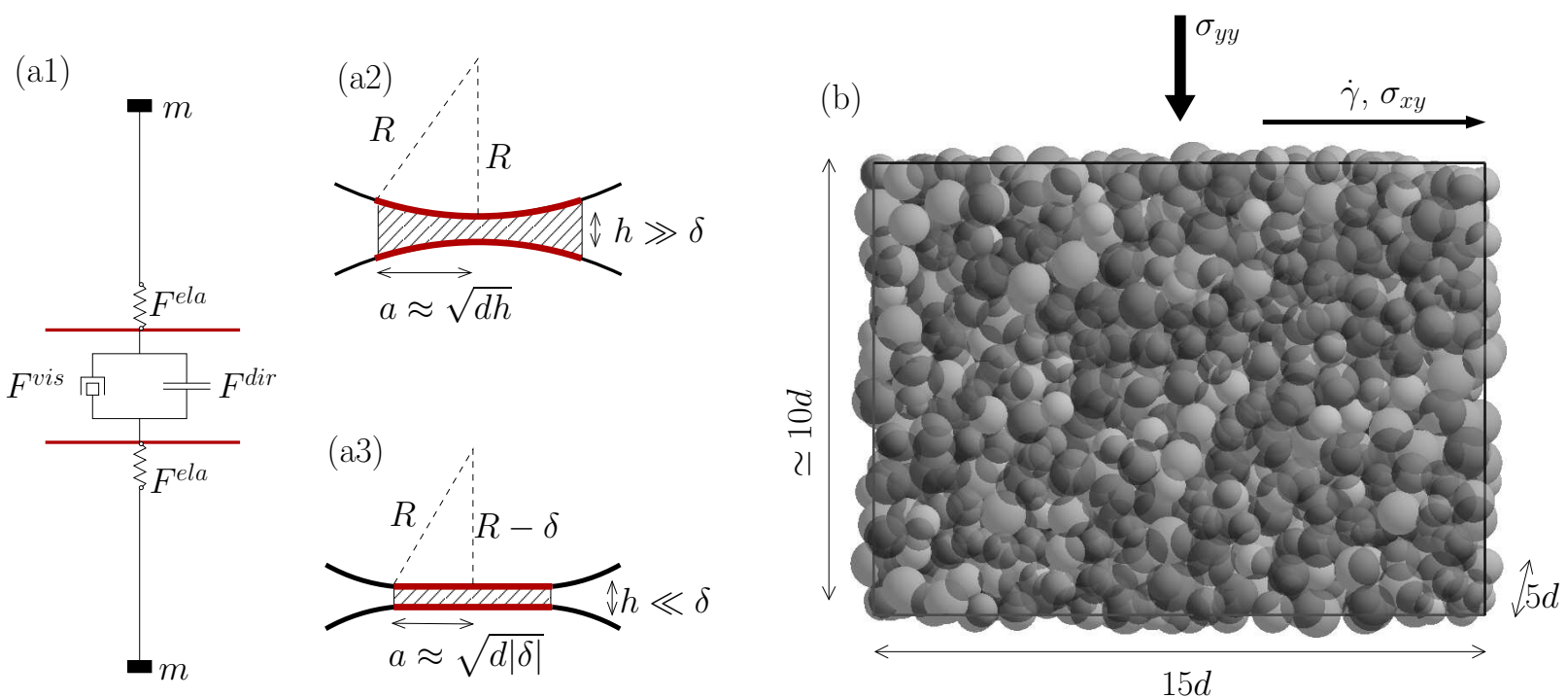

FIG. 1: (Color online) (a) Particle-fluid-particle interaction: (a1) the force $F^{\text {ela }}$ that deforms the surface elastically decomposes into the viscous force $F^{\text {vis }}$ and the direct particle-particle force $F^{d i r}$. The size $a$ of the transmitting force region is represented in two asymptotic limits: (a2) the Poiseuille limit for non-deflecting spheres, and (a3) the Hertz limit when the deflection $\delta$ is much larger than the gap width $h$. (b) Plane shear simulated using Soft Dynamics. 1000 particles are subjected to normal stress $\sigma_{y y}$ and shear stress $\sigma_{x y}$ in a periodic domain (Lees-Edwards boundary conditions [42]). The height of the cell fluctuates freely in order to keep $\sigma_{y y}$ constant.

gential sliding mode (Couette flow) and the twist mode. Thus, the normal relative motion should be responsible for the longest relaxation time. Let us therefore recall the expression of the normal viscous and elastic forces (see Appendix $\mathrm{A}$ ):

$$
\begin{aligned}
F_{n}^{v i s} & =\frac{3}{2} \pi \eta \frac{a^{4}}{h^{3}} \dot{h} \\
F_{n}^{e l a} & =a E \delta
\end{aligned}
$$

where $E$ is the Young modulus of the particle and $\delta$ the normal surface deflection. The size $a$ of the region that transmits most of the force basically depends on the distance between surfaces and on the flattening induced by their elastic deflections. If the elastic deflection is much smaller than the gap thickness, the surface shape is mostly spherical and the size $a$ is given by the classical lubrication approximation between two spheres: $a \approx \sqrt{d h}$ (Fig⿴囗十2). We refer to this asymptotic limit as the Poiseuille limit. Conversely, if the elastic deflection is much larger than the gap thickness, the particle surface elastically flattens over a region whose size is given by Hertz's theory: $a \approx \sqrt{d \delta}$ (Fig 1 a3). We refer to this asymptotic limit as the Hertz limit.

So far, two particles could not statically sustain any nonzero confining force, since the fluid would keep flowing out of the gap and the particle surfaces would correspondingly keep approaching although in a slower and slower motion. In practice, however, there is usually a typical distance $h_{0}$ below which particle surfaces interact not only via the fluid, but also through a direct particleparticle force. This interaction can have various physical origins, among which direct contacts between asperities of rough solid surfaces [22, 23, 24, 25] or repulsion between charged or polymeric surfactant molecules at the bubble or droplet surface [29]. Our present focus is not necessarily to capture any of the details of such effects, but rather to represent their common feature: a strong steric repulsion that works on the particle surfaces in parallel to the viscous force (Fig!1). For that purpose, we chose the following power law:

$$
F_{n}^{d i r}=F_{n}^{c o n f}\left(\frac{h}{h_{0}}\right)^{-\alpha}
$$

For $\alpha \gg 1$, this law captures our requirement for a strongly increasing repulsion as the gap becomes thinner than the steric length $h_{0}$, and a quickly vanishing repulsion for thicker gaps. Ideally, we would have liked to have an infinite $\alpha$, but as this function becomes more strongly non-linear, the critical time step for accurate simulations increases tremendously. A value of $\alpha=8$ was found as a good compromise between these two requirements. The force scale parameter is chosen as $F_{n}^{\text {conf }}=\sigma_{y y} d^{2}$. In this way, the surface-to-surface distances stabilize around $h_{0}$ when the material is subjected to a confining stress $\sigma_{y y}$. 


\section{COLLECTIVE RESPONSE TO A STEP SHEAR STRESS}

In this section, we use Soft Dynamics to study the response of the material to a sudden increase in shear stress in plane shear geometry, in the absence of gravity, with a fully periodic domain (Fig 10). The principle of the method is detailed in Appendix B. The typical experiment consists of two steps: ( $i$ ) the preparation of a dense compressed sample and (ii) the shear by itself.

First, particles are set in a loose and random configuration. They are then subjected solely to a designated target normal stress $\sigma_{y y}$. This makes the material denser, with closer particles, while the height of the periodic cell decreases. The material eventually reaches a static state where the confining stress is supported by some pairs of particles whose surface to surface distances are on the order of the steric length $h_{0}$. A correct simulation of this compression should include the long range multibody hydrodynamics interaction, while the particles are still in a loose configuration. However, we do not focus on the compression process and use it only as a way to obtain a close-packed configuration. In the second step, which is the prime focus of our paper, we subject the configuration to a (smoothened) step in shear stress from zero to a final value $\sigma_{x y}$. We then measure the time evolution of the nominal shear strain $\gamma$. More preciseley, the shear stress is continuously increased from zero to its final value within a short but finite time of the order of $t_{S t}$ (EqL2) as $\sigma_{x y}\left(1-e^{-\frac{t}{\beta t_{S t}}}\right.$ ) (see Fig. 2). Most of the results are obtained with $\beta=1$. Different values $(\beta=0.5$ and $\beta=2$ ) will be used to probe the influence of the rate of the initial shear stress increase.

We perform a series of such numerical experiments for materials with various steric lengths $h_{0}$, subjected to various levels of shear stress $\sigma_{x y}$. The parameters of the system are summarized in Tab. II expressed in units of particle size $d$ for the length, normal stress $\sigma_{y y}$ for the stress and Stokes time $t_{S t}$ for the time. The grains have a mass, but we set the inertial time much smaller than the Stokes time, so that the dynamics of the system is mostly driven by viscous interactions. The reduced normal stress $\kappa=\sigma_{y y} / E$ applied to the particles is much smaller than unity so that the elastic deformations would be much smaller than the particle size. We complete the current section by describing the resulting collective behavior in our Soft Dynamics simulations, which we will then analyse in terms of the internal dynamics in Section $\mathrm{V}$

\section{A. The role of the steric length}

In the first test we subject materials with different steric lengths $h_{0}$ to a similar constant shear stress $\sigma_{x y}=0.5 \sigma_{y y}$ (Fig. 2a). For the longest steric distance, $h_{0} / d=10^{-1}$, the shear strain increases almost steadily. In contrast, for the shortest distance $h_{0} / d=10^{-3}$, the
TABLE II: Properties of the simulated systems: inertial time $t_{i}$, reduced applied normal stress $\kappa$, steric distance $h_{0}$ and shear stress $\sigma_{x y}$.

\begin{tabular}{lccc}
\hline \hline$\frac{t_{i}}{t_{S t}}$ & $\kappa=\frac{\sigma_{y y}}{E}$ & $\frac{h_{0}}{d}$ & $\frac{\sigma_{x y}}{\sigma_{y y}}$ \\
\hline 0.1 & $10^{-3}$ & $8.10^{-4} \rightarrow 10^{-1}$ & $0.05 \rightarrow 1$ \\
\hline \hline
\end{tabular}

material first deforms very slowly. After this creep phase, it starts to flow steadily. During both the creep and the flow phases, $\gamma(t)$ is mostly linear (Fig. 20). Except for the very begining of the creeping phase, this behavior is not sensitive to the typical time of increase of the stress (Fig. 2 $\mathrm{b}$, inset). We define the typical duration $\Delta$ of the creep phase using a geometrical construction that searches for the intersection between these two lines, as plotted in linear coordinates. Note that prior to the creep phase, there is another very short phase, during which the shear deformation quickly increases up to a small level. This corresponds to the immediate elastic deformation of the particles induced by the increase in shear stress.

Fig. 3 shows the value of $\Delta$ for materials with different steric lengths $h_{0}$. The creep duration $\Delta$ naturally increases when $h_{0}$ gets smaller. However, as long as the steric length is larger than roughly $10^{-2} d, \Delta$ is small: only few times longer than the Stokes time. In contrast, it increases strongly for smaller $h_{0}$.

\section{B. Varying the step shear stress}

In the second test, a material with a steric length $h_{0}=$ $2.10^{-1} d$ was subjected to varying step shear stress values (Fig. 4). It appears that below a critical shear stress threshold, the material creeps and then does not seem to flow, but rather reaches a static equilibrium within a finite shear strain smaller than $100 \%$. Above this shear stress threshold, here found to be roughly $\sigma_{x y}=0.2 \sigma_{y y}$, the material creeps and then flows. One might imagine that the response delay in the creep phase before the onset of flow should be shorter if the applied constant shear stress is higher. Surprisingly, as can be seen from curves D-G of Fig. 4, i.e., with applied shear stresses that differ by a factor of 5 , it seems that the duration of the creep phase remains essentially constant although the transition from creep to flow becomes sharper for larger values of the applied shear stress.

\section{MICROSCOPIC ORIGIN OF THE OBSERVED RELAXATION TIME}

Let us now interpret the macroscopic behaviors observed in the previous section in terms of the dynamics of two particles in relative motion. When the material is sheared, neighbouring particles need to move towards 

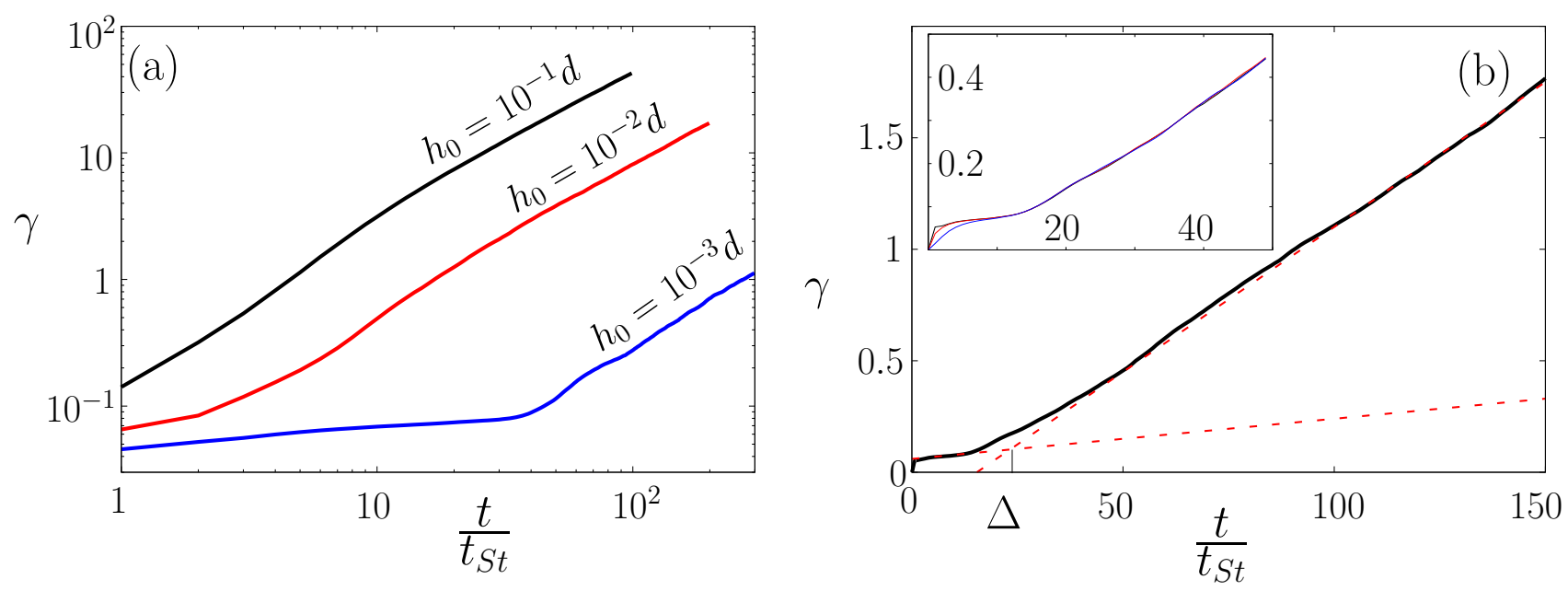

FIG. 2: (Color online) Time evolution of the shear strain $\gamma$ induced by a rapid increase in shear stress $\sigma_{x y}$ from 0 to $0.5 \sigma_{y y}$ between $t=0$ and $t=t_{S t}$. (a) Materials with a different value of the steric length $h_{0}$. (b) Procedure to determine the typical duration $\Delta$ of the creeping phase, here for $h_{0} / d=2.10^{-3}$; the dashed lines correspond to the linear fit of $\gamma(t)$ in the creep phase and in the flow phase; (Inset) for the same matrial, different rates of initial stress increase: $\beta=0.1$ (black), 0.5 (red) and 2 (blue) (see introduction of Section IV).

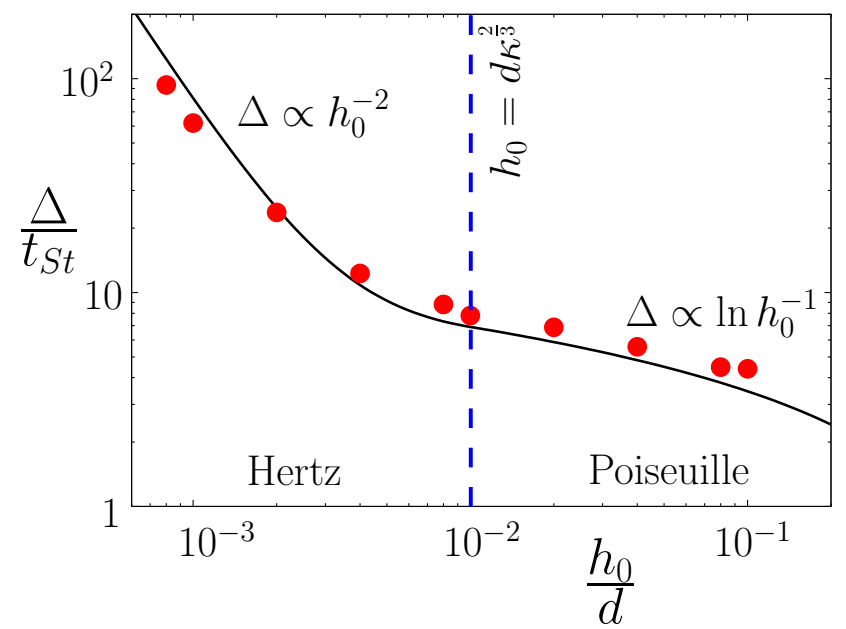

FIG. 3: (Color online) Macroscopic relaxation time $\Delta$, estimated as shown on Fig. 2p, as a function of the steric length $h_{0}$. Circles denote results from simulations, while the curve corresponds to 3 times the separation time $t^{\text {sep }}$ for two grains as defined by Eq. (8). The third data point $\left(h_{0} / d=2.10^{-3}\right)$ corresponds to curve F in Fig. 4 .

and away from each other. This relative motion includes sliding, rotation and normal approach and separation. As shown in Appendix $\mathrm{A}$, the normal viscous friction is the highest, which implies that this mode of motion should be the slowest. As a result, the material lies most of the time in a situation where interparticle contacts can be viewed as frictionless as far as sliding is concerned, with only normal forces acting to bring particles into contact or away from each other in a slow motion. Hence, we focus

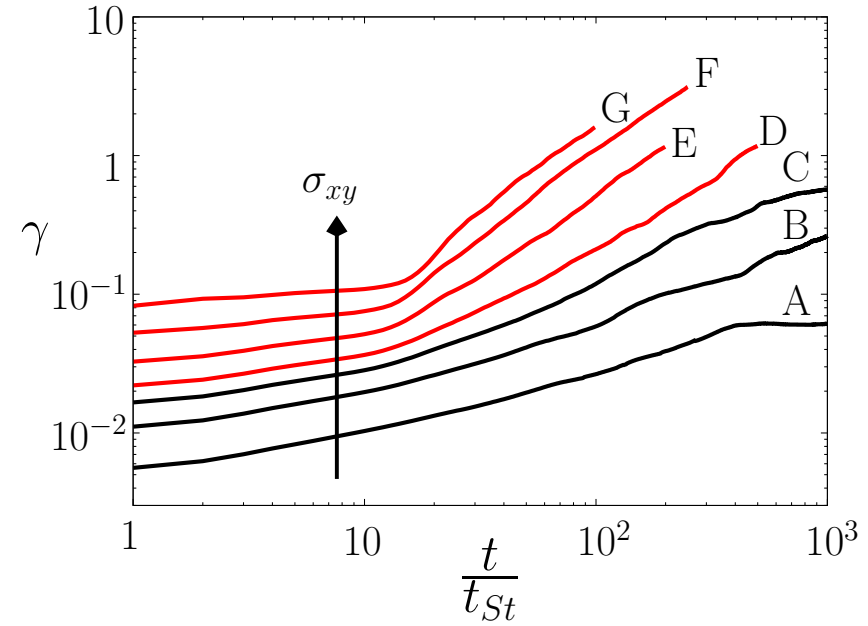

FIG. 4: (Color online) Time evolution of the shear strain $\gamma$ induced by establishing (from $t=0$ to $t=t_{S t}$ ) a constant shear stress (for larger $t$ ). Each curve corresponds to a different value of the final shear stress: $\sigma_{x y} / \sigma_{y y}=0.05(\mathrm{~A})$, 0.1 (B), 0.15 (C), 0.2 (D), 0.3 (E), 0.5 (F) and 1.0 (G). Red (resp. black) curves denote tests in which the material eventually flows (resp. does not flow). The same material (with $h_{0}=2 \cdot 10^{-3}$ ) was used for each run. Curve F corresponds to one of the experiments considered in Fig. 3

on the sole normal mode for interpreting the macroscopic observations.

The duration of the normal separation process depends on the initial distance between surfaces, on the viscous friction and on the typical force transmitted by the fluid in the gap. The typical initial distance between the sur- 
faces can be estimated as the steric distance $h_{0}$. As the step in shear stress is applied to the dense material, the initial viscous friction depends on whether the interaction is initially in the Poiseuille regime $\left(\delta<h_{0}\right)$ or in the Hertz limit $\left(\delta>h_{0}\right)$. Initially, the typical force transmitted within a pair of particles scales with $\sigma_{y y} d^{2}$. Hence, the interaction lies in the Hertz limit when $h_{0} / d<\kappa^{\frac{2}{3}}$.
Let us assume that, when the shear stress is applied, the typical transmitted force is still of the order of $\sigma_{y y} d^{2}$. Then, the time $t^{s e p}$ for two particles to separate is such that $\int_{t=0}^{t^{\text {sep }}} \sigma_{y y} d^{2} d t=\int_{h=h_{0}}^{h_{\text {sep }}} \frac{3}{2} \pi \eta \frac{a^{4}}{h^{3}} d h$, where the dimension of the force-transmitting region is $a=\sqrt{d h}$ in the Poiseuille limit and $a=\sqrt{d|\delta|}$ in the Hertz limit. Hence:

$$
\frac{t^{s e p}}{t_{S t}}= \begin{cases}\frac{1}{2} \ln \left(\frac{h_{\text {sep }}}{h_{0}}\right) & \text { (Poiseuille, } \left.\frac{h_{0}}{d} \gg \kappa^{\frac{2}{3}}\right), \\ \frac{1}{4}\left[\kappa^{\frac{4}{3}}\left(\frac{d}{h_{0}}\right)^{2}-1\right]+\frac{1}{2} \ln \left(\frac{h_{\text {sep }}}{d \kappa^{\frac{2}{3}}}\right) & \left(\text { Hertz, } \frac{h_{0}}{d} \ll \kappa^{\frac{2}{3}}\right) .\end{cases}
$$

An arbitrary value of $h^{s e p}$ has to be defined at the end of the separation process. Basically, it should be of the order of $d$. But the time of separation is only slightly depending on it. In the Poiseuille limit, the time of separation is at the order of the stokes time, with almost no effect by the small steric length $h_{0}$. In the Hertz limit, in contrast, it is strongly dependent on $h_{0}$ as well as on the reduced normall stress $\kappa=\frac{\sigma_{y y}}{E}$. When applied to the system simulated in the previous section $\left(\kappa=10^{-3}\right)$, this simple separation time $t^{s e p}$ captures the scaling behavior of the creep time $\Delta$ (Fig 3). The data are quantitatively well represented by the function $b t^{s e p}$ involving a fit parameter $b \approx 3$. This constant is likely to rely on the details of the contact network (number of coordination, heterogeneities, anisotropy...) and may also slightly depend on the geometrical definition of $\Delta$ (Fig 2 $\mathrm{b})$. The salient result is that, surprisingly enough, this very simple model with only one interaction and a single force scale $\sigma_{y y} d^{2}$ captures the macroscopic delay observed: both $t^{\text {sep }}$ and $\Delta$ scales with $\ln h_{0}^{-1}$ while the interaction is initially in the Poiseuille regime $\left(h_{0}>\kappa^{\frac{2}{3}}=10^{-2}\right)$, and with $h_{0}^{-2}$ if the interaction is initially in the Hertz regime (see Fig. 3).

However, this model does not account for the secondary effect of the shear stress we observed in Fig. 4, Basically, one could expect that under a larger applied shear stress, the typical tensile pairwise normal force would increase. Accordingly, one should observe a shorter separation time. Fig. 4 reveals that the creeping time appears to be essentially independent of the applied shear stress. This may have some connection, although probably not direct, with the counterintuitive effect highlighted in 32] concerning the crossover between the Poiseuille and the Hertz regimes: there exists an optimal tensile force that minimises the time of separation between two particles. This optimal force corresponds to the transition between the Hertz and the Poiseuille regimes. Thus, it depends on the gap $h$ as: $F^{o p t} \approx E d^{2}\left(\frac{h}{d}\right)^{\frac{3}{2}}$ (see 32] for more details). Increasing the force then brings the system further into the Hertz regime, which strongly increases the viscous friction and eventually results in a slower separation.

\section{CONCLUSION}

The distinction between the flowing and non-flowing states of many materials is critical for determining failure. The current paper focuses on studying the micromechanical origins of this phenomenon in relation to ideal materials of closed-packed elastic particles in a viscous fluid. Using Soft Dynamics simulations, we show that when subjected to a step shear stress, this material can exhibit a long creep phase before a steady flow state sets in. The typical creep time seems to rely on an internal relaxation process: the normal separation of two particles, which is driven by the applied stress and resisted by the viscous friction. This internal relaxation time thus depends on (i) the applied stress, (ii) the particle stiffness and (iii) the steric length $h_{0}$ below which particle surfaces interact directly.

The direct surface-to-surface interaction enables the material to statically sustain a confining stress, and whether or not the steric length is shorter than the typical elastic deflection strongly affects the scaling behavior of the viscous friction. On one hand, the relaxation time is not very long in the Poiseuille regime (large $h_{0}$ and/or very stiff particles) as its scales with $\ln \left(h_{0}^{-1}\right)$ and is thus only slightly longer than the Stokes time. On the other hand, the relaxation time can be very long in the Hertz limit (small $h_{0}$ and/or soft particles) as it then scales with $h_{0}^{-2}$ (see the estimation in table 【).

The long aging process corresponding to the Hertz limit should be a common characteristic of various immersed particulate materials like granular pastes, emulsions and foams. We expect that the soft bubbles and droplets would reach the Hertz regime for relatively low confining stresses. For the solid grains, a rough estimate can be performed according to Hertz's elasticity which defines the Hertz limit for $h_{0}<d\left(\frac{\sigma_{y y}}{E}\right)^{\frac{2}{3}}$. Assuming that the steric length $h_{0}$ is determined by a surface rough- 
ness of amplitude $0.1 \mu \mathrm{m}$, that the grain size is $d \simeq 1 \mathrm{~mm}$ and that their Young modulus is $E \simeq 10^{10} \mathrm{~Pa}$, then the normal stress required to reach the Hertz regime would be on the order of $\sigma_{y y} \simeq 10^{4} \mathrm{~Pa}$. Note, however, that these materials involve further modes of interaction such as solid friction, interfacial rheology and adhesion, which would (i) induce other time scales and (ii) affect the hydrodynamics within the gap and thus the expression of the viscous friction which the aging process relies on. In future works, we hope to include some such detailed interactions within our methodology.

\section{Acknowledgments}

Financial supports for this research from the Australian Government's Flagship Collaboration Fund through the CSIRO Flagship Cluster on Subsea Pipelines and from the French Government through the Agence Nationale de la Recherche (ANR-05-BLAN-0105-01) are appreciated.

\section{APPENDIX A: LUBRICATION BETWEEN ELASTIC SURFACES}

The viscous flow between between two close elastic spheres is not a simple problem. However, simple scaling formulae can be deduced considering that only a small portion of the surface transmits the force (size $a$ ), and that its radius of curvature is large $(a \ll R)$. Then, the hydrodynamical interaction is similar to that which develops between two flat parallel disks of radius $a$ separated by $h$. The low aspect ratio of the gap, $\frac{h}{a} \ll 1$ favors flows in the Stokes regime.

A simple way to get the scaling formulae for the viscous force and torque is to consider the average shear strain rate $\dot{\epsilon}$ in the liquid, which scales like $\frac{v^{s}}{h}$ for a Couette flow and like $\frac{3}{2} \frac{v^{s}}{h} \frac{\pi a^{2}}{2 \pi a h}$ for a Poiseuille flow. On the one hand, the power dissipated by the viscous flow in a gap of a volume $\pi a^{2} h$ scales with $\eta \dot{\epsilon}^{2} a^{2} h$. On the other hand, this power is equal to $F^{v i s} v^{s}$ for a relative velocity $v^{s}$ and to $\Gamma^{v i s} w^{s}$ for a relative angular velocity $w^{s}$. This leads to the expressions of the viscous forces and torques in Fig. 5. Note that the viscous force and torque can be expressed through the viscous friction tensors $Z$ and $w$ :

$$
\begin{array}{ll}
\vec{F}^{\text {vis }}=Z \cdot \vec{v}^{s} & \text { with } Z=\zeta \vec{n} \otimes \vec{n}+\lambda(I-\vec{n} \otimes \vec{n}) \\
\vec{\Gamma}^{\text {vis }}=W \cdot \vec{w}^{s} & \text { with } W=a^{2} Z,
\end{array}
$$

where $\vec{n}$ is the unit vector normal to the surface, $I$ the unity tensor and $\otimes$ the standard cross product.

The elastic forces and torques can also be estimated in a simple way by following the Hertz theory: the elastic strain $\epsilon$ concerns mostly a small region of volume $a^{3}$. It scales according to $\frac{\delta}{a}$ for both the normal and the tangential deflections represented by $\delta$, and as $\frac{a \theta}{a}$ for a twist angle $\theta$. The total elastic energy, $E \epsilon^{2}$, therefore scales like $E a^{3}\left(\frac{\delta}{a}\right)^{2}$ for deflections and according to $E a^{3} \theta^{2}$ for twists. The differentiation of these energies with respect to $\delta$ and $\theta$ gives the scaling for the elastic forces and torques expressed in Fig. 5. where all numerical constants are set to unity for the sake of simplicity.

\section{APPENDIX B: SOFT DYNAMICS METHOD}

We have developed the Soft Dynamics method to simulate large samples of closely packed particles with elastolubricated interaction 32, 33. In the present work, the particles are inertial so that their motion is deduced from the forces and torques they experience according to Newton's law, as in the standard discrete element methods:

$$
\left\{\begin{array}{l}
m_{i} \overrightarrow{\vec{X}}_{i}=\vec{F}_{i}^{e x t}+\sum_{j} \vec{F}_{i j}^{e l a} \\
J_{i} \vec{\Omega}_{i}=\vec{\Gamma}_{i}^{e x t}+\sum_{j}\left\{\vec{\Gamma}_{i j}^{e l a}+\vec{r}_{i j} \times \vec{F}_{i j}^{e l a}\right\} .
\end{array}\right.
$$

$m_{i}$ and $J_{i}$ are respectively the mass and the inertia moment of particle $i, \vec{X}_{i}$ and $\vec{\Omega}_{i}$ its position and its rotation velocity. $\vec{F}_{i}^{e x t}$ and $\vec{\Gamma}_{i}^{e x t}$ are possible external force and torque that the particle experiences, which are both taken as zero in the present study. The sums run on each particle $j$ interacting with $i$. The force $\vec{F}_{i j}^{e l a}$ and the torque $\vec{\Gamma}_{i j}^{\text {ela }}$ come from the elastic deflection and twist of the particle surfaces, and $\vec{r}_{i j}$ is the vector from the center of $i$ to the interaction point.

The uniqueness of the Soft Dynamics approach is to include the dynamics of the elastic deflection $\vec{\delta}_{i j}$ and twist angle $\vec{\theta}_{i j}$ so that the force and torque balances are satisfied for each interaction. $\vec{\delta}_{i j}$ and $\vec{\theta}_{i j}$ can be deduced from the difference between the particle velocities, $\vec{X}_{i}$ and $\vec{\Omega}_{i}$, and the relative translational and rotational velocities of particle surfaces, $\vec{v}_{i j}^{s}$ and $\vec{w}_{i j}^{s}$. Compatibility then requires to have:

$$
\left\{\begin{array}{l}
\vec{\delta}_{i j}=\vec{X}_{j}+\vec{\Omega}_{j} \times \vec{r}_{j i}-\left[\vec{X}_{i}+\vec{\Omega}_{i} \times \vec{r}_{i j}\right]-\vec{v}_{i j}^{s} \\
\vec{\theta}_{i j}=\vec{\Omega}_{j}-\vec{\Omega}_{i}-\vec{w}_{i j}^{s}
\end{array}\right.
$$

For each of the interactions, the relative velocities $\vec{v}_{i j}^{s}$ and $\vec{w}_{i j}^{s}$ are given by the force/torque balances:

$$
\left\{\begin{array}{l}
\vec{F}_{i j}^{e l a}=\vec{F}_{i j}^{v i s}+\vec{F}_{i j}^{d i r} \\
\vec{\Gamma}_{i j}^{e l a}=\vec{\Gamma}_{i j}^{v i s}+\vec{\Gamma}_{i j}^{d i r} .
\end{array}\right.
$$

and the fact that the viscous force/torque are proportional to them through the friction tensors $Z_{i j}$ and $W_{i j}$ (see App. A): 


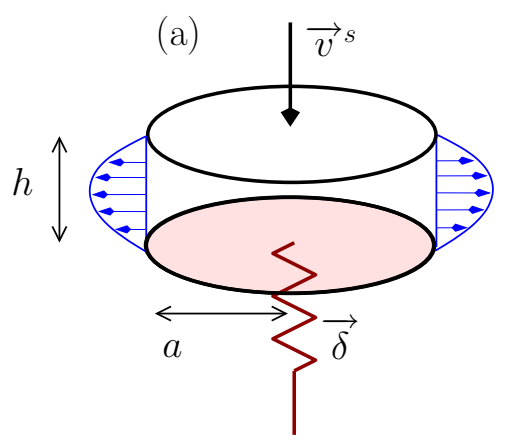

(b)

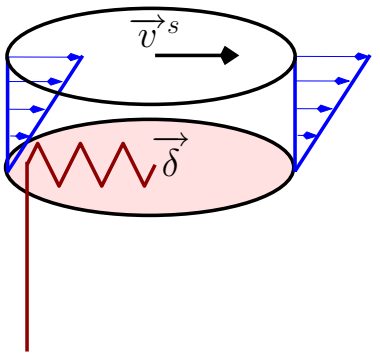

$$
\begin{aligned}
& \vec{F}^{\text {vis }}=\zeta \vec{v}^{s} \\
& \zeta=\frac{3}{2} \pi \eta{\frac{a^{4}}{h^{3}}}^{\text {a }} \overrightarrow{\vec{F}^{\text {ela }}=a E}
\end{aligned}
$$
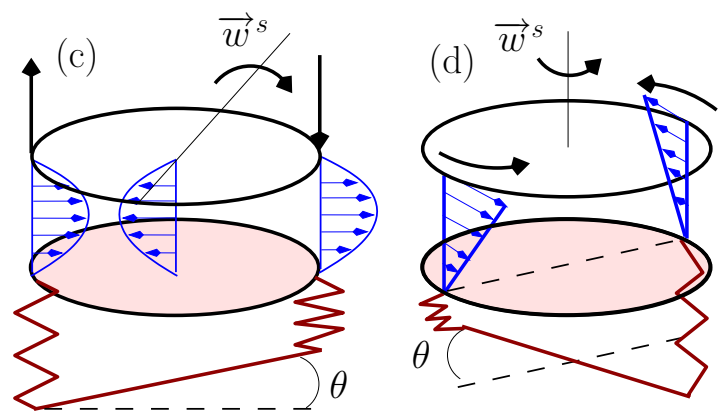

$$
\begin{aligned}
& \vec{\Gamma}^{v i s}=a^{2} \zeta \vec{w}^{s} \\
& \vec{\Gamma}^{e l a}=a^{3} E \vec{\theta}
\end{aligned}
$$$$
\vec{\Gamma}^{v i s}=a^{2} \lambda \vec{w}^{s}
$$

FIG. 5: (Color online) Scheme of the elasto-hydrodynamical interaction implemented in Soft Dynamics, including four modes of relative motion between particles with a surface-to-surface distance of $h$. Distance $a$ is the size of the transmitting force region (see Fig $1 \mathrm{a}$ ). The blue arrows designate the flow velocity profile and the red spring the elastic deformation of the bulk material. The corresponding expression of viscous and elastic forces/torques are as follows. (a) The normal approach induces a Poiseuille flow and an associated viscous friction $\zeta$ (also valid for a normal separation). (b) The tangential sliding induces a Couette flow and an associated viscous friction $\lambda$. (c) Normal rotation (locally, the flow is like a Poiseuille flow). (d) Parallel rotation (locally, the flow is like a Couette flow).

$$
\left\{\begin{array}{l}
\vec{v}_{i j}^{s}=Z_{i j}^{-1} \cdot\left[\vec{F}_{i j}^{e l a}-\vec{F}_{i j}^{d i r}\right] \\
\vec{w}_{i j}^{s}=W_{i j}^{-1} \cdot\left[\vec{\Gamma}_{i j}^{e l a}-\vec{\Gamma}_{i j}^{d i r}\right] .
\end{array}\right.
$$

The second order equations (B2) are integrated using a standard predictor-corrector scheme, while the first order equations (B3) are integrated using a simple Euler scheme. The time step of integration is a fraction of the shortest time scale in the system, namely $t_{M}=\frac{\eta}{E}$. Through careful examination we found that a time step of five time smaller than $t_{M}$ is short enough.
Since we do not include long range hydrodynamical interactions, it is sensible to introduce a cut-off distance: here two particle interact only if their surface to surface distance is smaller than $0.25 d$. The size of the transmitting force region $a$ is then being interpolated between the two asymptotic limits of the Poiseuille and the Hertz models, as a way to get a continuous transition. The interpolation we choose is: $a=\sqrt{d(h+|\delta|)}$. Note that different choices of interpolation are possible, although this should have no incidence except in the vicinity of the crossover between both scaling regimes.
[1] R. Höhler and S. Cohen-Addad, J. Phys. : Cond. Mat. 17, R1041 (2005).

[2] N. Denkov, S. Tcholakova, K. Golemanov, K. Ananthpadmanabhan, and A. Lips, Soft Matter 5, 3389 (2009).

[3] L. Bécu, S. Manneville, and A. Colin, Phys. Rev. Lett. 96, 138302 (2006).

[4] S. Derkach, Advances in Colloid and Interface Science 151, 1 (2009).

[5] L. Bocquet, A. Colin, and A. Ajdari, Phys. Rev. Lett. 103, 36001 (2009).

[6] F. Yilmaz and M. Gundogdu, Korea-Australia Rheology Journal 20, 197 (2008).

[7] J. Stickel and R. Powell, Annu. Rev. Fluid Mech. 37, 129 (2005).

[8] P. Coussot, Rheometry of pastes, suspensions, and granular materials (Wiley-Interscience, 2005).
[9] P. Sollich, F. Lequeux, P. Hébraud, and M. Cates, Phys. Rev. Lett. 78, 102020 (1997).

[10] P. Richard, M. Nicodemi, R. Delannay, P. Ribiere, and D. Bideau, Nature Materials 4, 121 (2005).

[11] G. Marty and O. Dauchot, Phys. Rev. Lett. 94, 015701 (2005).

[12] P. Coussot, Soft Matter 3, 528 (2007).

[13] H. Barnes, J. Non Newtonian Fluid Mech. 70, 1 (1997).

[14] F. da Cruz, F. Chevoir, D. Bonn, and P. Coussot, Phys. Rev. E 66, 051305 (2002).

[15] P. Møller, J. Mewis, and D. Bonn, Soft Matter 2, 274 (2006).

[16] A. Grillet, R. Rao, D. Adolf, S. Kawaguchi, and L. Mondy, J. Rheol. 53, 169 (2009).

[17] T. Narumi, H. See, A. Suzuki, and T. Hasegawa, J. Rheol. 49, 71 (2005). 
[18] H. Wyss, K. Miyazaki, J. Mattsson, Z. Hu, D. Reichman, and D. Weitz, Phys. Rev. Lett. 98, 238303 (2007).

[19] R. Iverson, Journal of Geophysical Research-Earth Surface 110, F02015 (2005).

[20] M. Pailha, M. Nicolas, and O. Pouliquen, Phys. Fluids 20, 111701 (2008).

[21] G. K. Batchelor, An introduction to fluid dynamics (Cambridge University Press, Cambridge, 1967).

[22] J. Jenkins and M. Koenders, Geophys. Mag. 7, 13 (2005).

[23] O. Vinogradova and G. Yakubov, Phys. Rev. E 73, 45302 (2006).

[24] L. Yang, J. Seddon, T. Mullin, C. Del Pino, and J. Ashmore, J. Fluid Mech. 557, 337 (2006).

[25] T. Divoux and J. Geminard, Phys. Rev. Lett. 99, 258301 (2007).

[26] M.-D. Lacasse, G. S. Grest, and D. Levine, Phys. Rev. E 54, 5436 (1996).

[27] M. Durand and A. Stone, Phys. Rev. Lett. 97, 226101 (2006).

[28] L. Sagis, Phys. Rev. Lett. 98, 66105 (2007).

[29] S. Tabakova and K. Danov, J. Coll. Inter. Sci. (2009).

[30] S. Besson, G. Debregeas, S. Cohen-Addad, and R. Höhler, Phys. Rev. Lett. 101, 214504 (2008).

[31] N. Denkov, S. Tcholakova, K. Golemanov, and A. Lips,
Phys. Rev. Lett. 103, 118302 (2009).

[32] P. Rognon and C. Gay, Eur. Phys. J. E 27, 253 (2008).

[33] P. Rognon and C. Gay, Submitted to Eur. Phys. J. E (2009).

[34] F. da Cruz, F. Lechenault, and O. Dauchot, in Powders and Grains 2005, edited by R. Garcia-Rojo, H. J. Herrmann, and S. McNamara (A.A. Balkema, Leiden, The Netherlands, 2005), pp. 7-11.

[35] GDR MiDi, Euro. Phys. J. E 14, 341 (2004).

[36] Y. Forterre and O. Pouliquen, Annu. Rev. Fluid Mech. 40, 1 (2008).

[37] C. Cassar, M. Nicolas, and O. Pouliquen, Phys. Fluids 17, 103301 (2005).

[38] D. Doppler, P. Gondret, T. Loiseleux, S. Meyer, and M. Rabaud, J. Fluid Mech. 577, 161 (2007).

[39] C. Campbell, J. Fluid Mech. 539, 273 (2005).

[40] E. Clent, J. Rajchenbach, and J. Duran, Europhys. Lett. 30, 7 (1995).

[41] J. Duran, Sables, poudres et grains (Eyrolles Sciences, Paris, 1997).

[42] A. W. Lees and S. Edwards, J. Physics C: Solid State Phys 5, 1921 (1972). 